\title{
Characterization of Glomerulonephritis and Pyelonephritis using Ultrasonography
}

\author{
MEM Garelnabi ${ }^{1}$, Ibtisam Abdulallah ${ }^{1}$, Elsafi Ahmed Abdulla ${ }^{1}$, Abdoelrahman Hassan A. B. ${ }^{1}$, Mohamed Adam ${ }^{2}$ \\ ${ }^{1}$ Sudan University of Science and Technology, College of Medical Radiological Science, Khartoum, Sudan \\ ${ }^{2}$ King Khalid University, Colleges of Applied Medical Sciences, Radiology Department, ABHA, Saudi Arabia
}

\begin{abstract}
Ultrasound (US) is one modality used to assess renal infections, because it's a simple, Produces image in real time, less expensive, accurate method and well accepted by patient in comparison to other modality. This study aimed to determine the characterization of incidence of renal infections by US, in Khartoum, Wad Madani, Elmanagil hospitals and Elkramit family health center, in urology department. An analytical study on the sonographic pattern of renal infections in 234 person from January 2014 to May 2016. Ultrasound scanning has been carried out, using a curve linear probe with a frequency of 3.5 to 5 MHz. this study reveals that female was mostly affected by glomerulonephritis and pyelonephritis rather than male with male to female ratio of 1:1.6 and 1:2 respectively. Flank pain in $82.4 \%$ associated with glomerulonephritis while $75 \%$ of pyelonephritis showed ill-defined corticomedullary differentiation. In conclusion Ultrasonographic characteristics in addition to medical laboratory test can be used in a multiple linear regression equation to diagnose the patients affect by kidney infection with a classification accuracy of $96 \%$.
\end{abstract}

Keywords: Ultrasound, glomerulonephritis, pyelonephritis, discriminant, kidney, UTI

\section{Introduction}

Kidneys lie behind the peritoneum on the posterior abdominal wall on either side of the vertebral column, each kidney has outer cortex and inner medulla, the medulla is composed of about twelve renal pyramids, the renal papilla projecting medially [1]. Normal Sonographic Appearances of the kidney parts when demonstrated in its long axis, the capsule is an echogenic; pre renal fat is highly echogenic. The renal cortex is homogeneous, textured and poorly echogenic. The cortex is equal to or less echogenic than the normal liver. The medulla consists of pyramids which are anechoic structures; renal sinus is the most echogenic portion of the adult kidney. This echogenic area is called the central echo complex zone. In the non-hydrated state the renal pelvis is collapsed as compare to the parenchymal organ echogenicity Renal sinus $>$ spleen $>$ liver $\geq$ renal cortex $>$ renal medulla [2].

Brandt et al. (1982) assessed normal renal dimensions using Ultrasound. Their study confirms that the accuracy and reliability of sonographic assessment of renal dimensions, with patients in the prone position, the mean right renal length was $10.74 \mathrm{~cm}( \pm 1.35 \mathrm{SD})$ and the mean left renal length was $11.10 \mathrm{~cm}( \pm 1.15 \mathrm{SD})$. A prospective sample demonstrated the mean depth (ventral-dorsal dimension) to be approximately $4.5 \mathrm{~cm}$ when the transducer was angulated for the lie of the kidney [3].

The predisposing factors of renal infections occur via the ascending route, usually caused by contaminants from the intestinal tract, instrumentation, stasis calculi, and vesicoureteral reflux. And the other infection result from intravenous drug abuse, tuberculosis and in immune compromised patients [1]. These infection causes in lower urinary tract which include the bladder which called cystitis and urethra and those of the upper tract that include the kidneys, renal pelvis, and ureters. Are diagnosed nephritis (Glomerulonephritis GN and pyelonephritis PN). [4].
Sonographically PN most commonly normal but you may find a focal area of enlargement and decreased echogenicity involving a renal parenchyma, loss of corticomedullary differentiation with increase in renal size. These features accompany the generalized changes of acute pyelonephritis. The focal area involved can degenerate into an abscess. [2] Most sonographic appearance of nephritis is an area of increased echogenicity in the parenchyma of the affected kidney. [5]

Glomerulonephritis is a disease of the renal glomeruli associated with various systemic diseases such, as systemic lupus erythematous. Sonographically, the affected kidneys may range from normal to markedly enlarge size. The echo pattern of the cortex is altered with medullary sparing and may be normal, Hypoechoic or Hyperechoic. With treatment the kidneys revert to normal size and echo pattern [6].

In renal Parenchymal Diseases, the echogenicity of renal parenchyma and cortex increased in all types of renal parenchymal diseases. [7].

\section{Material and Methods}

A total of 234 patient in urology department participated in this study, which has taken place between January 2014 to May 2016, Khartoum hospital, Madani hospital, Almangil hospital and Elkraymit family health center. A data collection sheet was designed to include the demographic data and clinical history of every patient. Informed consent was taken from every patient. The clinical history of every patient had been reported. Ethics and research was approved by Committee of faculty of radiological sciences and medical imaging. (SUST).

\section{Ultrasound Examination}

Renal ultrasound examination has performed with Mindary DC-7 and My lab 60 ultrasound machines with 3.5-5 MHz

\section{Volume 5 Issue 8, August 2016}




\section{International Journal of Science and Research (IJSR) \\ ISSN (Online): 2319-7064 \\ Index Copernicus Value (2013): 6.14 | Impact Factor (2015): 6.391}

probe is typically used to scan the kidney. The kidneys assessed in the transverse and coronal plane. Optimal patient positioning varies; supine and lateral decubitus positions often suffice, although oblique and occasionally prone positioning may be necessary (e.g., obese patients). Usually, a combination of subcostal and intercostal approaches is required to evaluate the kidneys fully; the upper pole of the left kidney may be particularly difficult to image without a combination of approaches. [8]

\section{Result and Discussion}

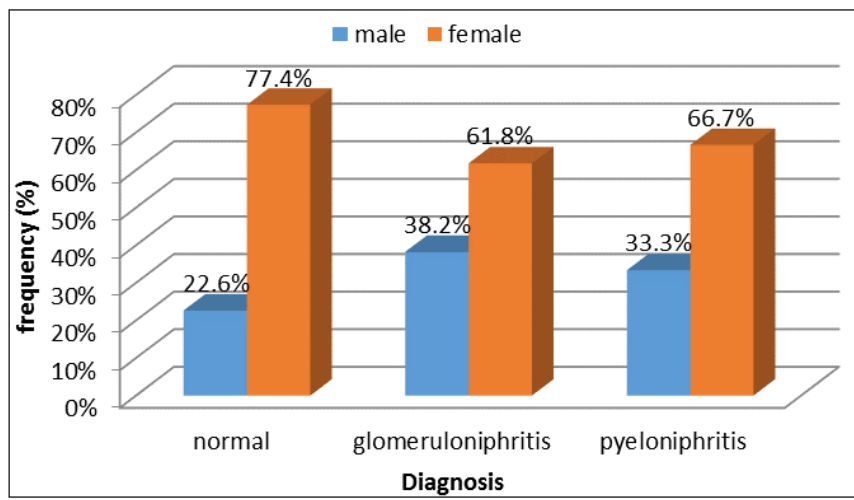

Figure 1: A bar graph illustrate the \% frequency distribution of patient diagnosis according to their gender.

A total of 234 patients were included in this study 106 were normal cases $(22.6 \%$ male and $77.4 \%$ female) 128 patients had renal infections; 68 diagnosed with glomerulonephritis (38.2\% males and $61.8 \%$ females) 60 with pyelonephritis (33.3\% males and 66.7 females); the number of female were higher than that of male with a male to female ratio for glomerulonephritis and pyelonephritis of $1: 1.6$ and $1: 2$ respectively; which mean that females were more susceptible for renal infection than males. The patient age ranged from (11 to 80) years also indicates that infection appears in a wide spectrum of range which might be attributed to hygiene problem. The common presenting symptoms were flank pain which appear more in patients with glomerulonephritis (Figure 2).

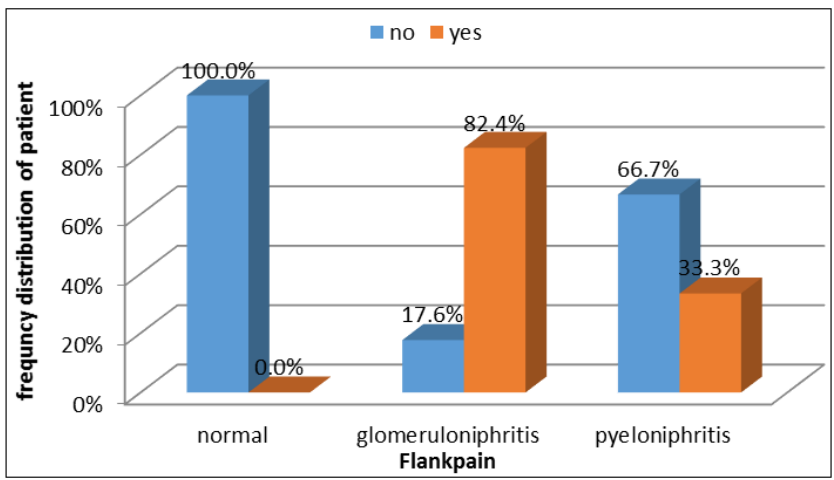

Figure 2: Illustrate the frequency distribution of flank pain

Significant differences between the patients with normal kidneys and those affected by glomerulonephritis and pyelonephritis in case of medical laboratory test, as well as Sonographic results at $(\mathrm{p}=0.05)$ using t-test. Sonographic appearance that showed significant differences from the normal kidney appearance includes: kidney length and cortical thickness for the right and left kidney; where incase of length the infected kidney appears larger than the normal one mostly attributed to infection condition, on other hand cortical thickness appear significantly more thinner than that of normal patient. The rest of the variable concerning the Sonographic measurement outcome showed inconclusive results they include kidney width, thickness and size for both kidneys. Laboratory investigation revealed significant differences at $(\mathrm{p}=0.05)$ between patient with normal kidneys and infected one concerning RBC, WBC, Protein, and Pus cells where all these tests gives significantly higher values than patient with normal kidneys (Table 1,2)

Table 1: The mean \pm Standard deviation of the laboratory measured variable including the t-test values and the probability level (significant $<0.05$ ).

\begin{tabular}{|c|c|c|c|c|c|}
\hline \multicolumn{2}{|c|}{ Variables } & Mean & SD & $t$ & Sig. (2-tailed) \\
\hline \multirow{2}{*}{$\mathrm{RBC}$} & Normal & 0.4 & 0.9 & \multirow{2}{*}{8.96} & \multirow{2}{*}{$\underline{0.000}$} \\
\hline & Abnormal & 24.5 & 19.4 & & \\
\hline \multirow{2}{*}{ WBC } & Normal & 6230.8 & 1113.5 & \multirow{2}{*}{16.5} & \multirow{2}{*}{$\underline{0.000}$} \\
\hline & Abnormal & 13415 & 2908.6 & & \\
\hline \multirow{2}{*}{ protein } & Normal & 0.0 & 0.0 & \multirow{2}{*}{12.9} & \multirow{2}{*}{$\underline{\mathbf{0 . 0 0 0}}$} \\
\hline & Abnormal & 1.7 & 0.9 & & \\
\hline \multirow{2}{*}{ Urea } & Normal & 21.8 & 5.4 & \multirow{2}{*}{1.29} & \multirow{2}{*}{0.200} \\
\hline & Abnormal & 24.0 & 10.6 & & \\
\hline \multirow{2}{*}{ Pus cells } & Normal & 1.3 & 1.1 & \multirow{2}{*}{15.4} & \multirow{2}{*}{$\underline{\mathbf{0 . 0 0 0}}$} \\
\hline & Abnormal & 50.1 & 22.8 & & \\
\hline Keratin & Normal & 0.9 & 0.2 & 7.1 & $\underline{0.000}$ \\
\hline
\end{tabular}

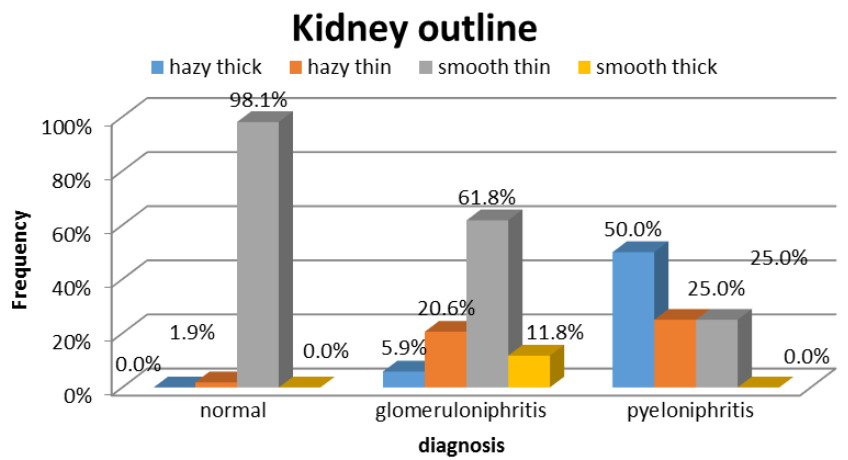

Figure 3: \% frequency distribution of Ultrasonographic appearances of kidney outline from normal kidneys and infected one (glomerulonephritis and pyelonephritis).

Ultrasound scanning showed that kidney outline of $50 \%$ of pyelonephritis were hazy thick and $25 \%$ were hazy thin, where for glomerulonephritis $61.8 \%$ were smooth thin similar to normal while $20.6 \%$ showed hazy thin outline; this means pyelonephritis affected the outline of the kidneys more than glomerulonephritis because the later-on affected calyces rather than medulla area (Figure 3).

Table 2: The mean \pm Standard deviation of the sonographic characteristics variable including the t-test values and the probability level (significant $<0.05$ )

\begin{tabular}{|c|c|c|c|c|c|}
\hline \multicolumn{2}{|c|}{ Variables } & Mean & $\begin{array}{c}\text { Std. } \\
\text { Deviation }\end{array}$ & $t$ & Sig. (2-tailed) \\
\hline \multirow{2}{*}{ RKL } & Normal & 8.3 & 0.9 & \multirow{2}{*}{6.16} & \multirow{2}{*}{$\underline{0.000}$} \\
\hline & Abnormal & 9.6 & 1.3 & & \\
\hline \multirow{2}{*}{ RKW } & Normal & 4.1 & 0.7 & \multirow{2}{*}{0.75} & \multirow{2}{*}{0.453} \\
\hline & Abnormal & 4.0 & 0.6 & & \\
\hline RKT & Normal & 2.9 & 0.3 & 0.07 & 0.944 \\
\hline
\end{tabular}


International Journal of Science and Research (IJSR)

ISSN (Online): 2319-7064

Index Copernicus Value (2013): 6.14 | Impact Factor (2015): 6.391

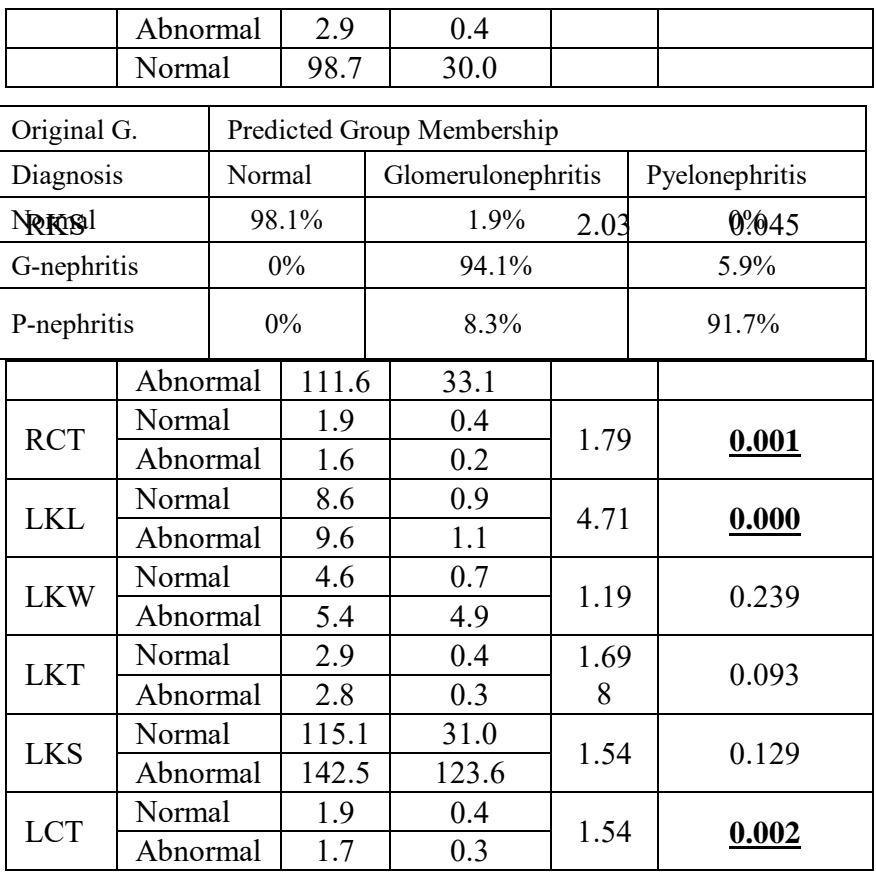

\section{Abbreviations:}

$R K L:$ Right Kidney Length

RKW: Right Kidney Width

RKT: Right Kidney Thickness

RKS: Right Kidney $\underline{\text { Size }}$

RCT: Right cortical Thickness

(Same for left kidney).

For echotexture $58.8 \%$ of glomerulonephritis reveals hypoechogenic textures due to the presence of fluid as a result of infection, while pyelonephritis ranged from hperechogenic to hyopechogneic as well as isoechogenic depending on the stage of the disease (Figure 4). While corticomedullary differentiation (CMD) effect appear mostly in case of pyelonephritis in which $75 \%$ of the kidneys appear with ill-defined or poor differentiation between the medulla and the cortices (Figure 5)

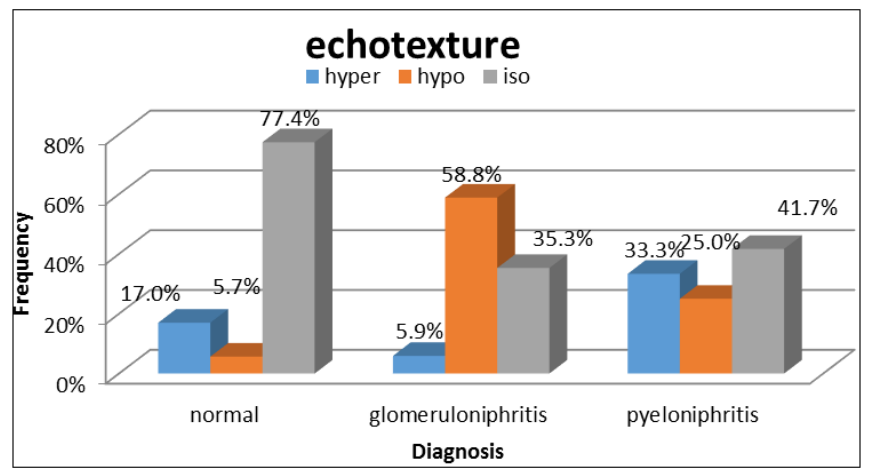

Figure 4: Echotexture of normal kidneys and the affected kidneys by glomerulonephritis and pyelonephritis.

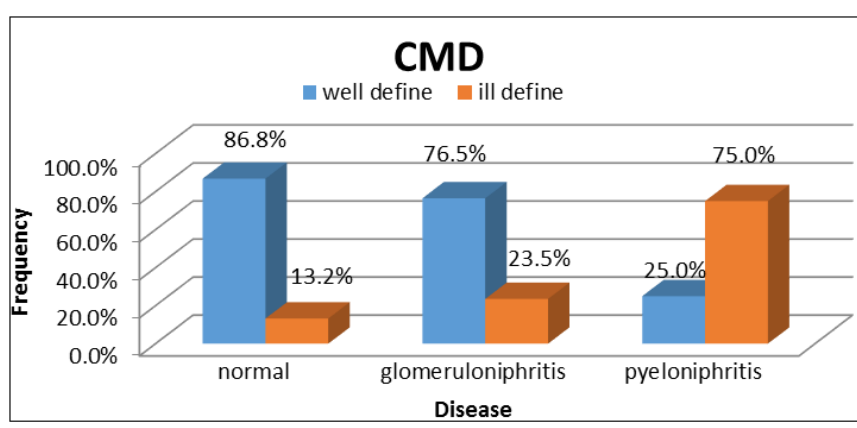

Figure 5: Ultrasonographic appearance of corticomedullary differential of normal kidneys and the affected kidneys by glomerulonephritis and pyelonephritis.

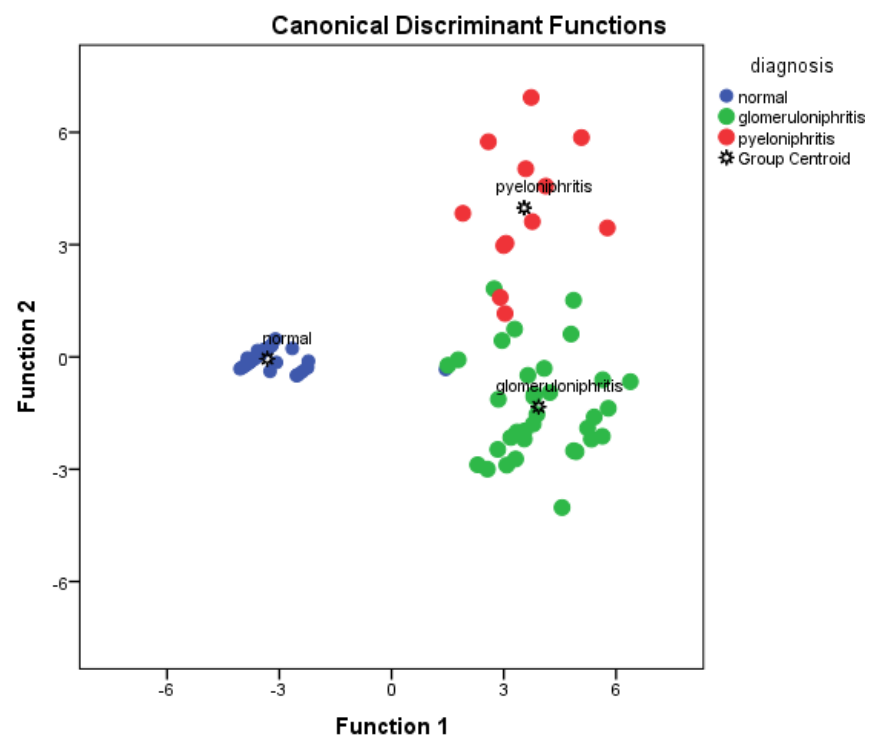

Figure 6: Scatter plot depicted the classification of kidney status as normal, glomerulonephritis and pyelonephritis using discriminant analysis. The classification score using linear discriminant analysis was $96 \%$ as shown in table 3 .

Table 2. Classification score table related the accuracy of the predicted groups to the original group- Original group

\section{Conclusions}

Renal infections corresponding to glomerulonephritis and pyelonephritis can be diagnosed by ultrasonography according to it is manifestations which represented by echotexture, CMD and outline of the kidney profile as well as kidney dimensions measurement like kidney length and cortical thickness, in addition to the medical laboratory test as an indicator for the presence of renal infection. The characteristics (presence of flank pain, RBC, WBC, CMD, kidney length and cortical thickness) that identify the renal infections type from the normal kidney were used in this study to identify each group objectively using linear discriminant analysis, where the classes grouped together in a class when there is a minimum between class variance while it shows maximum differences between class variance (Figure 6).

From linear discriminant analysis three multiple linear regression were generated (Eq1, Eq2 and Eq3) where the variable that correlated well with the classes (normal,

\section{Volume 5 Issue 8, August 2016}




\section{International Journal of Science and Research (IJSR) \\ ISSN (Online): 2319-7064}

Index Copernicus Value (2013): 6.14 | Impact Factor (2015): 6.391

glomerulonephritis and pyelonephritis) can be used to classify the patient as one of these categories according to the higher vote objectively.
Medical physics, ultrasound and Nuclear Medicine researches. Now he is lecturer at SUST also (2016).

Normal $=81.222-(-7.858 \times$ Flankpain $)+(-0.185 \times \mathrm{RBC})+$

$(0.001 \times \mathrm{WBC})+(15.00 \times \mathrm{CMD})+(12.500 \times$ Kidney length $) \times$ $(17.717 \times$ cortical thickness $) \quad \underline{\text { (Eq.1) }}$

Glomerulonephritis $=2.308+(-.185 \times$ Flankpain $)+(.002 \times \mathrm{RBC})+$ $(17.231 \times \mathrm{WBC})+(12.459 \times \mathrm{CMD})+(17.919 \times$ Kidney length $)+(-$ $100.147 \times$ cortical thickness)

(Eq. 2)

Pyelonephritis $=95.584-(-4.745 \times$ Flankpain $)+(-.065 \times \mathrm{RBC})+$ $(.002 \times \mathrm{WBC})+(18.987 \times \mathrm{CMD})+(12.154 \times$ Kidney length $) \times$ (16.595 $\times$ cortical thickness $)$ (Eq. 3)

\section{References}

[1] Dean D. Buirwin Institute of Diagnostic Medical Ultrasound. Obstetrical ultrasound 2002,(1-3)197-214

[2] R.S.Snell, Clinical Anatomy By System ,Lippincott Williams \&Wilkins 2007.

[3] D Brandt, H.L.Neiman, M.J. Dragowski, W. Bulawa, G. Claycamp : Ultrasound assessment of normal renal dimensions.J Ultrasound Medl :49-52,1982.

[4] William V. Raszka, Omar Khan, Sonographic assessment of pyelonephritis, October 2005, Kfar Saba. Isr Med Assoc J. 2007 Oct;9(10):729-31 Rathaus V(1), Werner Medical Imaging, Acute focal nephritis

[5] Brown, P. (2003) Ultrasound in Diffuse Renal Disease. British Medical Ultrasound Society Bulletin, 11, 3035.http://ult.sagepub.com/content/11/4/30.full.pdf.

[6] Carol M ;Rumack Stephanie R,Wilson J William,Charboneau,Deborah Levie, Diagnostic ultrasound imaging .sonographic technique ,2011,4th edi,9-321.

[7] Awadia Gareeballah, Moawia Gameraddin1, Hago Mustafa, Sultan Alshabi, Fath Elerahman Alagab, Jumaa Tamboul, Suliman Salih Sonographic Findings in Renal Parenchymal Diseases at Sudanese Open Journal of Radiology, 2015, 5, 243-249.

\section{Author Profile}

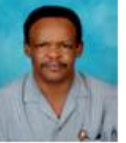

Dr. Mohamed Elfadil Mohamed Gar-elnabi (Sudan) awarded the B. Sc. in Radiotherapy and Nuclear Medicine (1987) and M.Sc. in Radiation Therapy (2000-SUST) and PhD degree in Medical Physics (Natal University-South Africa) in 2007. During 1996-2012 he has been working as lecturer as well as Associate Prof. at SUST department of Radiation therapy. Also he has been active in Computerized Texture Analysis, Radiotherapy-Oncology, Ultrasound and Nuclear Medicine researches.

Mr. Abdoelrahman Hassan Ali Bakry (Sudan) received the (B.Sc.) and (M.Sc.-1) in radiotherapy technology from College of Medical radiological Science, Sudan University of Science and Technology in 2013 and 2015 respectively. M.Sc.-2 (student) Diagnostic Radiology Technology, National University (Sudan)-2016. During 2013 up to date, he is staying in College of Medical radiological Science, Sudan University of Science and Technology, Radiology Department, Antalya Medical Center and Elnileen Diagnostic Medical Center; also he has been active in Computerized Texture

Analysis, Radiotherapy-Oncology, and Diagnostic Radiology, 